\title{
Utility of Hyperbaric Oxygen Therapy for Acute Acoustic Trauma: 20 years' Experience at the Japan Maritime Self-Defense Force Undersea Medical Center
}

\author{
Morihiko Oya ${ }^{1}$ Yutaka Tadano ${ }^{1}$ Yasuhiro Takihata ${ }^{1}$ Fumitaka Ikomi ${ }^{2}$ Tetsuji Tokunaga ${ }^{1}$ \\ ${ }^{1}$ Department of Medical Research, Japan Maritime Self-Defense Force \\ Undersea Medical Center, Yokosuka, Japan \\ 2 Department of Research Institute, National Defense Medical \\ College, Tokorozawa, Japan \\ Address for correspondence Morihiko Oya, MD, Research Division, \\ Experimental Department, Maritime Self-Defense Force Undersea \\ Medical Center, Taura Minatocho, Yokosuka, Kanagawa 237-0071, \\ Japan (e-mail: sphz9rx9@carol.ocn.ne.jp).
}

Int Arch Otorhinolaryngol 2019;23:e408-e414.

\begin{abstract}
Introduction Acute acoustic trauma, which is a kind of sensorineural hearing loss, is caused by acoustic overstimulation. Hyperbaric oxygen therapy (HBOT) is reported to be effective against acute acoustic trauma.

Objective We aimed to evaluate the efficacy of HBOT against acoustic hearing loss based on our 20 years of experience with such cases.

Methods Patients who were treated with HBOT for acute acoustic trauma between April 1997 and August 2017 were evaluated in this study. Thirty-five patients with a mean age of $25.7 \pm 9.2$ (range: $16-48$ ) years were included. Thirty-nine out of 70 ears (35 patients) were damaged. We investigated the initial level of hearing loss; the extent to which hearing recovered; subjective symptoms, such as tinnitus and aural fullness; and the treatment administered.

Results The planned HBOT was completed in 37 of 39 ears. Twenty-six of the 37 ears

Keywords

- HBOT

- sensorineural deafness

- tinnitus (70.2\%) displayed improved hearing, and 31 of the 37 ears (83.9\%) exhibited symptom improvement. Twenty-three $(76.7 \%)$ and 26 (86.7\%) of the 30 ears treated with steroids demonstrated improvements in hearing and subjective symptoms, respectively.

Conclusion A combination of HBOT and steroids should be considered as a treatment for acute acoustic trauma in cases involving symptoms such as tinnitus and aural fullness.
\end{abstract}

\section{Introduction}

Acute acoustic trauma, which is a kind of sensorineural hearing loss, is caused by acoustic overstimulation. Noise exposure can cause temporary or permanent hearing loss. ${ }^{1}$ Severe inner ear damage, which can occur at critical sound intensity levels, is considered to result in permanent hearing loss. $^{2-4}$ In addition, acute acoustic trauma can occur after short periods of acoustic overstimulation. This type of hearing loss might be reversible, at least in part, if an adequate management strategy is initiated early enough. Even in acute acoustic trauma, however, repeated exposure to critical

received

June 25, 2018

accepted

March 17, 2019
DOI https://doi.org/

10.1055/s-0039-1688433. ISSN $1809-9777$. sound intensity levels over long periods of time can lead to permanent hearing loss. On the other hand, hearing impairment due to repeated acoustic overstimulation at relatively low levels is called noise-induced hearing loss, and it often leads to permanent hearing loss. Patients with acute acoustic trauma sometimes exhibit symptoms such as tinnitus and aural fullness.

Compared with people in other occupations, the members of the Japan Self-Defense Forces (JSDF) tend to be exposed to high noise levels and intense sounds more often during their work. This can lead to hearing loss, tinnitus, and/or aural fullness, which can interfere with their performance in

Copyright @ 2019 by Thieme Revinter

Publicações Ltda, Rio de Janeiro, Brazil

License terms

c) $(1) \$$ 
missions. Therefore, acute acoustic trauma is recognized as an occupational condition among JSDF members.

Various treatment modalities have been used to treat acute acoustic trauma, with steroid therapy and hyperbaric oxygen therapy (HBOT) being the most commonly used of these treatments. Some antioxidants have also been found to be effective. However, the optimal medical therapy for acute acoustic trauma remains unclear, and there is a need to formally evaluate the therapeutic effects of HBOT on acute acoustic trauma. In addition, there have been few studies of the changes in the symptoms of acute acoustic trauma, such as tinnitus and aural fullness, induced by HBOT. Harada reported cases of acute acoustic trauma involving JSDF members. ${ }^{5}$ In this study, we reviewed the cases of patients with acute acoustic trauma who were treated with HBOT at the Maritime Self-Defense Force (MSDF) Undersea Medical Center during a 20-year period and evaluated whether HBOT is useful for treating acute acoustic trauma.

\section{Methods}

\section{Patients}

Patients who were treated with HBOT for acute acoustic trauma at the MSDF Undersea Medical Center between April 1997 and August 2017 were included in this study. Thirty-five patients were diagnosed with acute acoustic trauma during this period. We retrospectively reviewed their records. The patients had a mean age of $25.7 \pm 9.2$ years (range: 16-48 years). Thirty-two patients were male, and none of them had a history of hearing impairment before their acute acoustic trauma. Nineteen patients were MSDF members, 11 were Ground Self-Defense Force members, 4 were National Defense Academy students, and one was an Air Self-Defense Force member.

\section{Hearing Tests}

We reviewed the following items:

- The cause of the acute acoustic trauma

- The initial hearing loss level

- The initial subjective symptoms

- The interval from the onset of the condition to the start of treatment

- The duration of the HBOT

- The recovery of hearing after the HBOT

- The amelioration of subjective symptoms after the HBOT

- The presence/absence of steroid use

To estimate the initial degree of hearing loss, hearing tests based on the pure-tone average (PTA; the mean of the values for $500 \mathrm{~Hz}, 1,000 \mathrm{~Hz}$, and $2,000 \mathrm{~Hz}$ ) and high pure-tone average (HPTA; the mean of the values for $4,000 \mathrm{~Hz}$ and $8,000 \mathrm{~Hz}$ ) were performed. Absolute hearing improvement was defined as the difference in the hearing test results obtained using an audiometer before and after treatment. Relative hearing improvement, that is, the percentage recovery, was defined as the absolute hearing improvement divided by the initial degree of hearing loss multiplied by
100. The clinical outcomes of the patients were classified into 3 grades:

Grade 1: complete recovery; that is, hearing was restored to within $<20 \mathrm{~dB}$.

Grade 2: partial recovery; that is, the mean loss had improved by $\geq 10 \mathrm{~dB}$ at the follow-up.

Grade 3: unchanged; that is, the observed improvement was $<10 \mathrm{~dB}$ or the patient's hearing had deteriorated.

The recovery of hearing and the amelioration of subjective symptoms were evaluated at $>3$ weeks after the HBOT.

\section{Hyperbaric Oxygen Therapy}

To treat patients with acute acoustic trauma with HBOT, we used the methods outlined in U.S. Navy treatment table 5 (TT5) or treatment table 9 (TT9). The procedures described in TT5 and TT9 are shown in - Fig. 1. The methods outlined in TT5 and TT9 involve the consumption of 3,000 L and 2,500 L of oxygen, respectively. The unit pulmonary toxic doses (UPTD) for TT5 and TT9 are 334 and 270, respectively.

\section{Statistical Analyses}

Fisher exact test was used to compare the recovery grades of the TT5 and TT9 groups, as well as those of the HBOT with steroid therapy and HBOT alone groups. The Mann-Whitney U-test was used to compare the recovery percentage values of the TT5 and TT9 groups, as well as those of the HBOT with
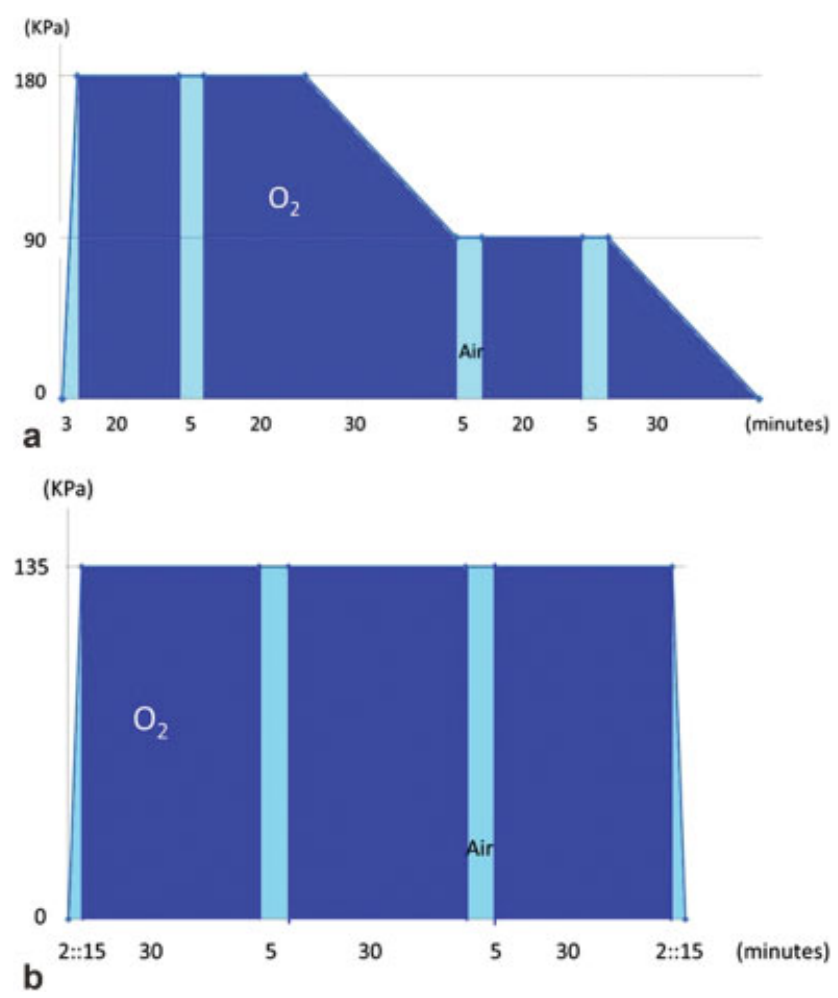

Fig. 1 (a) U.S. Navy Treatment Table 5 (TT5). The procedure for TT5 is shown below. After compression to $180 \mathrm{kPa}$, the patient alternates between $\mathrm{O}_{2}$ breathing and air breathing. After decreasing to $90 \mathrm{KPa}$, the patient alternates between air breathing and $\mathrm{O}_{2}$ breathing. This takes 2 hours and 15 minutes. (b) U.S. Navy Treatment Table 9 (TT9). The procedure for TT9 is shown below. After compression to $135 \mathrm{kPa}$, the patient alternates between $\mathrm{O}_{2}$ breathing and air breathing. This takes 1 hour and 45 minutes. 
Table 1 The patients' characteristics at baseline

\begin{tabular}{|c|c|c|c|}
\hline & TT5 & TT9 \\
\hline \multicolumn{2}{|l|}{ Age } & $\begin{array}{l}23.9 \pm 10.7 \\
(16-48 \text { years })\end{array}$ & $\begin{array}{l}27.7 \pm 8.4 \\
(17-45 \text { years })\end{array}$ \\
\hline \multirow[t]{2}{*}{ Gender } & male & 7 & 25 \\
\hline & female & 0 & 3 \\
\hline \multicolumn{2}{|c|}{ Number of ears } & 7 & 30 \\
\hline \multicolumn{2}{|c|}{$\begin{array}{l}\text { Interval from onset } \\
\text { to beginning of } \\
\text { treatment (days) }\end{array}$} & $10.3 \pm 7.6$ & $27.8 \pm 53.7$ \\
\hline \multicolumn{2}{|c|}{$\begin{array}{l}\text { Treatment period } \\
\text { (days) }\end{array}$} & $6.5 \pm 1.1$ & $8.5 \pm 2.4$ \\
\hline \multicolumn{2}{|c|}{$\begin{array}{l}\text { Numbers of ears } \\
\text { with steroid } \\
\text { treatment }\end{array}$} & 6 & 24 \\
\hline
\end{tabular}

steroid therapy and HBO alone groups. P-values of $<0.05$ were regarded as statistically significant.

\section{Results}

\section{Patients' Characteristics and the Causes of Acute Acoustic Trauma}

Among the 35 acute acoustic trauma patients, the causes included: shooting training in 30 patients (85.7\%), training and work other than shooting training in 3 patients (8.6\%), and other causes not associated with work in the remaining 2 patients (5.7\%). Thirty-nine out of 70 ears ( 35 patients) were damaged. The maximum extent of the patients' hearing loss was $60.1 \pm 18.9 \mathrm{~dB}$ (frequency at which hearing loss was detected: 4,000 Hz: 18 ears, 2,000 Hz: 9 ears, 8,000 Hz: 8 ears, 1,000 Hz: 2 ears, and 3,000 Hz: 2 ears). The mean amount of hearing loss in the PTA test was $32.9 \pm 16.0 \mathrm{~dB}$ (range: $0-75.0 \mathrm{~dB}$ ), and the mean amount of hearing loss in the HPTA test was $48.4 \pm 18.7 \mathrm{~dB}$ (range: $15.0-92.5 \mathrm{~dB}$ ).

\section{Effects of HBOT on Hearing Loss}

Hyperbaric oxygen therapy was completed in 30 male (34 ears) and 3 female ( 3 ears) patients. Thus, 33 of the 35 patients (94.4\%) (37 of 39 ears) successfully completed the HBOT. All of them complained of tinnitus or aural fullness as subjective symptoms. The mean duration of the interval from
Table 3 The recovery grades of the 37 ears classified according to whether their subjective symptoms improved

\begin{tabular}{|l|l|l|}
\hline \multirow{2}{*}{} & \multicolumn{2}{|l|}{ Subjective symptoms } \\
\cline { 2 - 3 } & Improved & Not improved \\
\hline Grade 1 & 4 & 0 \\
\hline Grade 2 & 22 & 0 \\
\hline Grade 3 & 5 & 6 \\
\hline Total & 31 & 6 \\
\hline
\end{tabular}

There was a statistically significant correlation between the frequency of subjective symptom improvement and the recovery grade $(p<0.01$, Fisher exact test).

the onset of the condition to the start of HBOT was $24.5 \pm 48.5$ days. In the 7 ears that were treated using TT5, the mean duration of the treatment period was $6.5 \pm 1.1$ days. In the 30 ears that were treated using TT9, the mean duration of the treatment period was $8.5 \pm 2.4$ days. In the remaining 2 ears, barotrauma occurred during the HBOT, and the treatment could not be completed ( - Table $\mathbf{1}$ ).

Regarding the recovery grades of the 37 ears in which HBOT was completed, 4 (10.8\%) ears were classified as grade 1,22 (59.4\%) ears were classified as grade 2 , and the remaining 11 (29.7\%) ears were classified as grade 3 . So, the hearing loss was ameliorated in 26 of the 37 ears (70.2\%). The mean absolute hearing improvement was $15.6 \pm 16.3 \mathrm{~dB}$ (range: -5.0 to $56.7 \mathrm{~dB}$ ), and the mean relative hearing improvement was $40.9 \pm 29.5 \%$ (range: -13.0 to $91.9 \%$ ) according to the PTA tests. In the HPTA tests, mean absolute and relative hearing improvements of $20.4 \pm 20.3 \mathrm{~dB}$ (range: -2.5 to $77.5 \mathrm{~dB}$ ) and $37.7 \pm 32.7 \%$ (range: -10.0 to $93.0 \%$ ), respectively, were seen. Of the ears treated using TT5, 2 ears were classified as grade 2, and 5 ears were classified as grade 3 . Of the ears that were treated using TT9, 4 ears were classified as grade 1, 20 ears were classified as grade 2 , and 6 ears were classified as grade 3 . There was a significant difference between the hearing improvements achieved with TT5 and TT9 ( $p=0.016$, Mann-Whitney U-test) (-Table 2). The HPTA testing indicated that the recovery percentage achieved with TT9 was significantly greater than that achieved with TT5 ( $p=0.028$, Mann-Whitney U-test). Thirty-one of the 37 ears (83.8\%) showed improvements in their subjective symptoms (tinnitus or aural fullness) (-Table 3). Of the 31 ears whose subjective symptoms

Table 2 The recovery percentage and recovery grade achieved with each treatment table

\begin{tabular}{|l|l|l|l|l|l|l|l|l|l|}
\hline & \multicolumn{3}{|l|}{ Hearing loss before HBOT $(\mathrm{dB})$} & \multicolumn{2}{l|}{ Recovery percentage (\%) } & \multicolumn{3}{l|}{ Recovery grade } \\
\cline { 2 - 9 } & Max & PTA & HPTA & Max & PTA & HPTA & Grade 1 & Grade 2 & Grade 3 \\
\hline TT5 & $50.0 \pm$ & $19.6 \pm$ & $35.4 \pm$ & $17.7 \pm$ & $37.9 \pm$ & $17.1 \pm$ & 0 & 2 & 5 \\
& $17.5 \mathrm{~dB}$ & $11.7 \mathrm{~dB}$ & $19.1 \mathrm{~dB}$ & $17.1 \%$ & $29.6 \%$ & $25.9 \%$ & & 4 & \\
\hline TT9 & $63.4 \pm$ & $29.7 \pm$ & $51.4 \pm$ & $21.5 \pm$ & $41.7 \pm$ & $43.6 \pm$ & 4 & 20 & 6 \\
& $17.1 \mathrm{~dB}$ & $18.8 \mathrm{~dB}$ & $21.2 \mathrm{~dB}$ & $10.4 \%$ & $28.9 \%$ & $31.5 \%$ & & & \\
\hline
\end{tabular}

Abbreviations: HBOT, hyperbaric oxygen therapy; HPTA, high pure-tone average; PTA, pure-tone average.

There was no statistically significant difference in the recovery percentage in the PTA ( $p=0.738$, Mann-Whitney U-test) or the recovery percentage compared with the maximum extent of hearing loss $(p=0.3$, Mann-Whitney U-test) between the cases treated using TT5 and TT9. There were statistically significant differences in the percentage recovery in the HPTA ( $p=0.028$, Mann-Whitney U-test) and the recovery grade $(p=0.016$, Fisher exact test) between the cases treated using TT5 and TT9. 
improved, 6 (19.4\%) were treated using TT5, and the remaining 25 ears (80.6\%) were treated using TT9. In the cases treated using TT5 and TT9, the mean duration of the treatment period was 6.0 days and 8.6 days, respectively. Of the ears whose subjective symptoms improved, 4 ears were classified as grade 1, 22 ears were classified as grade 2 , and the remaining 5 ears were classified as grade 3 . All of the ears whose subjective symptoms did not improve were classified as grade 3 . There was a statistically significant correlation between the frequency of subjective symptom improvement and the extent to which hearing recovered ( $p<0.01$, Fisher exact test) ( - Table 3 ). In the patients whose subjective symptoms improved, the mean interval from the onset of the condition to the start of the HBOT was $26.2 \pm 54.7$ days, while in the patients whose symptoms did not improve, it was $14.7 \pm 12.0$ days.

\section{Effects of Steroid Treatment for Acute Acoustic Trauma}

Thirty $(81.1 \%)$ of the 37 ears were treated with steroids. Twenty-six $(86.7 \%)$ of the 30 ears had been treated with steroids at a previous hospital. Only 6 (23.1\%) of the 26 ears exhibited improvements in their hearing ability after the steroid treatment; thus, all of these steroid-treated patients were referred to our institution to undergo HBOT in the hope of achieving greater improvements in their hearing ability and subjective symptoms.

Among the 30 steroid-treated ears, 4 were classified as grade 1,19 were classified as grade 2 , and 7 were classified as grade 3 . So, 23 (76.7\%) of the 30 ears that were administered steroids showed improvements in their hearing ability. Their PTA and HPTA improved by $42.5 \pm 30.8 \%$ and $39.6 \pm 32.5 \%$, respectively ( $p=0.264,0.103$, Mann-Whitney U-test). Of the 7 ears that were not treated with steroids, 3 were classified as grade 2 , and 4 were classified as grade 3 . Their PTA and HPTA improved by $25.4 \pm 17.2 \%$ and $16.4 \pm 37.9 \%$, respectively. There were no significant differences in the recovery grade or recovery percentage between the ears treated with and without steroids ( - Table 4 ). Among the 7 ears treated using TT5, 6 (85.7\%) were administered steroids. Of these 6 ears, 2 were classified as grade 2 , and 4 were classified as grade 3 . Their PTA and HPTA improved by $42.6 \pm 32.4 \%$ and $24.5 \pm 25.9 \%$ ( $p=1.0$ and 0.134 , respectively; Mann-Whitney U-test) (-Table 4). Among the 30 ears that were treated using TT9, 24 (60.0\%) were administered steroids. Of these 24 ears, 4 were classified as grade 1,17 were classified as grade 2 , and 3 were classified as grade 3 . Their PTA and HPTA improved by $45.9 \pm 29.3 \%$ and $24.5 \pm 25.9 \%(p=0.156$ and 0.027, respectively; Mann-Whitney U-test) (-Table 4). Among the cases treated using TT5, there were no statistically significant differences in the percentage recovery compared with the maximum extent of hearing loss or in the percentage recovery in the PTA or HPTA ( $p=0.803,1.0$, and 0.134 , respectively; Mann-Whitney U-test) or the recovery grade ( $p=0.714$, Fisher exact test) between the ears treated with or without steroids (-Table 4 ).

Improvements in subjective symptoms were seen in 26 (86.7\%) of the 30 ears. However, there was no statistically significant difference in the frequency of subjective symptom improvement between the ears treated with or without steroids among all ears, the TT5-treated ears, or the TT9treated ears ( $p=0.316,0.143$, and 0.702 , respectively; Fisher exact test) (-Table 5). Of the 26 ears whose subjective symptoms improved, 4 were classified as grade 1,19 were classified as grade 2 , and the remaining 3 were classified as grade 3 . Steroid treatment did not ameliorate the subjective symptoms of 4 ears, all of which were classified as grade 3 . The hearing levels of 2 of the 4 ears whose subjective symptoms did not improve deteriorated, according to both PTA and HPTA testing, and the degree of hearing loss was unchanged in the remaining 2 ears.

Pulse corticosteroid therapy involving 500 mg methylprednisolone was administered to 12 of the 22 patients whose

Table 4 The recovery grade and recovery percentage of the ears treated with hyperbaric oxygen therapy with or without steroids

\begin{tabular}{|c|c|c|c|c|c|c|c|}
\hline & & \multicolumn{2}{|l|}{ Overall } & \multicolumn{2}{|l|}{ TT5 } & \multicolumn{2}{|l|}{ TT9 } \\
\hline & & \multicolumn{2}{|c|}{ Steroid therapy } & \multicolumn{2}{|c|}{ Steroid therapy } & \multicolumn{2}{|c|}{ Steroid therapy } \\
\hline & & $(+)$ & $(-)$ & $(+)$ & $(-)$ & $(+)$ & $(-)$ \\
\hline \multirow[t]{3}{*}{ Recovery (\%) } & Max & $23.0 \pm 20.4 \%$ & $11.5 \pm 18.5 \%$ & $20.6 \pm 16.7 \%$ & $0 \%$ & $21.5 \pm 21.6 \%$ & $13.8 \pm 19.5 \%$ \\
\hline & PTA & $42.5 \pm 30.8 \%$ & $25.4 \pm 17.2 \%$ & $42.6 \pm 32.4 \%$ & $33.30 \%$ & $45.9 \pm 29.3 \%$ & $23.8 \pm 18.4 \%$ \\
\hline & HPTA & $39.6 \pm 32.5 \%$ & $16.4 \pm 37.9 \%$ & $24.5 \pm 25.9 \%$ & $-10.00 \%$ & $48.3 \pm 31.3 \%$ & $7.90 \pm 9.87 \%$ \\
\hline \multirow[t]{3}{*}{ Recovery grade } & Grade 1 & 4 & 0 & 0 & 0 & 4 & 0 \\
\hline & Grade 2 & 19 & 3 & 2 & 0 & 17 & 3 \\
\hline & Grade 3 & 7 & 4 & 4 & 1 & 3 & 3 \\
\hline
\end{tabular}

Abbreviations: HPTA, high pure-tone average; PTA, pure-tone average, TT5, U.S. Navy treatment table 5; TT9, U.S. Navy treatment table 9. There was no statistically significant difference in the recovery percentage compared with the maximum extent of hearing loss or in the PTA or HPTA ( $p=0.767,0.264$, and 0.103 , respectively; Mann-Whitney U-test) or the recovery grade ( $p=0.099$, Fisher exact test) between the cases treated with and without steroids. Among the TT5-treated ears, there were no statistically significant differences in the recovery percentage compared with the maximum extent of hearing loss or in the PTA or HPTA ( $p=0.803,1.0$, and 0.134 , respectively; Mann-Whitney U-test) or the recovery grade ( $p=0.714$, Fisher exact test) between the cases treated with and without steroids. Among the TT9-treated ears, there was a statistically significant difference in the HPTA between the cases treated with and without steroids ( $p=0.027$, Mann-Whitney U-test). There was no statistically significant difference in the recovery percentage compared with the maximum extent of hearing loss or in the PTA ( $p=0.337$ and 0.156 , respectively; MannWhitney U-test) or the recovery grade ( $p=0.075$, Fisher exact test) between the cases treated with and without steroids. 
e412 Utility of Hyperbaric Oxygen Therapy for Acute Acoustic Trauma Oya et al.

Table 5 The 37 ears classified according to whether their subjective symptoms improved and whether steroid therapy was administered

\begin{tabular}{|l|l|l|l|l|l|l|l|}
\hline \multicolumn{2}{|c|}{} & \multicolumn{2}{|l|}{ Overall } & TT5 & \multicolumn{2}{l|}{ TT9 } \\
\cline { 3 - 8 } \multicolumn{2}{|c|}{} & $\begin{array}{l}\text { with } \\
\text { steroids }\end{array}$ & $\begin{array}{l}\text { without } \\
\text { steroids }\end{array}$ & $\begin{array}{l}\text { with } \\
\text { steroids }\end{array}$ & $\begin{array}{l}\text { without } \\
\text { steroids }\end{array}$ & $\begin{array}{l}\text { with } \\
\text { steroids }\end{array}$ & $\begin{array}{l}\text { without } \\
\text { steroids }\end{array}$ \\
\hline $\begin{array}{l}\text { Subjective } \\
\text { symptoms }\end{array}$ & Improved & 26 & 5 & 6 & 0 & 20 & 5 \\
\cline { 2 - 9 } & Not improved & 4 & 2 & 0 & 1 & 4 & 1 \\
\hline
\end{tabular}

Abbreviations: TT5, U.S. Navy treatment table 5; TT9, U.S. Navy treatment table 9.

There was no statistically significant difference in the frequency of subjective symptom improvement between the ears treated with and without steroids among all ears, the TT5-treated ears, or the TT9-treated ears ( $p=0.316,0.143$, and 0.702 , respectively; Fisher exact test).

Table 6 Evaluation of the effects of the type and dose of steroid therapy administered (including the presence/absence of methylprednisolone pulse therapy)

\begin{tabular}{|c|c|c|c|c|c|c|c|c|c|c|}
\hline & \multicolumn{10}{|c|}{ Administered steroid therapy } \\
\hline & \multirow{2}{*}{$\begin{array}{l}\text { mPSL pulse } \\
\text { therapy } \\
-\end{array}$} & \multicolumn{8}{|c|}{ Gradual dose reduction } & \multirow[t]{2}{*}{ Total } \\
\hline $\begin{array}{l}\text { Maximum steroid } \\
\text { dose }(\mathrm{mg})\end{array}$ & & $<30$ & $30-40$ & $40-50$ & $50-60$ & $60-70$ & $70-100$ & $100-150$ & $150-200$ & \\
\hline $\begin{array}{l}\text { Subjective symptoms } \\
\text { improved }\end{array}$ & 13 & 0 & 1 & 2 & 3 & 0 & 2 & 1 & 4 & 26 \\
\hline $\begin{array}{l}\text { Subjective symptoms } \\
\text { did not improve }\end{array}$ & 0 & 1 & 2 & 0 & 0 & 1 & 0 & 0 & 0 & 4 \\
\hline
\end{tabular}

Abbreviation: mPSL, methyl-prednisolone.

For non-methyl-prednisolone pulse therapy, the maximum dose of prednisolone is shown. The values in the bottom two rows represent numbers of ears.

subjective symptoms were ameliorated. The other 10 patients underwent steroid therapy involving gradual dose reduction, starting at a maximum prednisolone dose of $200 \mathrm{mg}$. The 4 patients who showed no improvements in their subjective symptoms after steroid treatment underwent steroid therapy involving gradual dose reduction, starting at a maximum prednisolone dose of $70 \mathrm{mg}$ (-Table 6 ).

\section{Discussion}

The optimal treatment for sudden sensorineural hearing loss (SSHL) is disputed. So, the treatment employed varies widely and often depends on the patient's geographic location. The treatment regimens administered (on either an inpatient or outpatient basis) for SSHL include antiviral agents; hemodilution agents; mineral, vitamin, and herbal preparations; batroxobin; carbogen; and HBOT. In addition, some otolaryngologists choose not to treat SSHL at all, citing the reported spontaneous recovery rates of 32 to $70 \%$. Severe acoustic overstimulation can cause the loss or mechanical fusion of the inner ear stereocilia, the loss of the adjacent supporting cells, or disruption of the organ of Corti. ${ }^{2}$ In addition to the direct damage inflicted on the inner ear stereocilia or adjacent supporting cells in the inner ear, capillary stenosis, a reduction in the partial pressure of oxygen in the inner ear, and metabolite consumption are included in the pathologies of acute acoustic trauma. ${ }^{6}$ Hyperbaric oxygen therapy is a potentially effective treatment for acute acoustic trauma. To achieve a consistent increase in perilymph oxygen content, which is the primary oxygen source for the intracochlear structures, the arterialperilymphatic oxygen concentration can be restored with HBOT. Ylikoski ${ }^{7}$ reported that HBOT achieved significantly greater average improvements in hearing levels and tinnitus than normobaric oxygen therapy, among patients with acute acoustic trauma. Specifically, they found that the PTA and HPTA improved in $~ 70 \%$ of patients.

In the present study, we observed that acute acoustic trauma was ameliorated in $70 \%$ of the cases treated with HBOT (including those involving partial responses). Ylikoski ${ }^{7}$ studied patients with acute acoustic trauma who were treated with HBOT within 3 to 4 days of symptom onset. On the other hand, at our institution the mean duration of the interval from the onset of acute acoustic trauma to HBOT was $\sim 24.4$ days. These results suggest that administering HBOT in combination with steroids might be an appropriate treatment for acute acoustic trauma, providing that it is administered within 3 to 4 weeks of symptom onset. Moreover, tinnitus and/or aural fullness were ameliorated in $\sim 80 \%$ of the patients. The mean interval from the onset of the condition until the initiation of HBOT was 26.2 days among the patients whose symptoms improved, while it was 14.7 days among the patients whose symptoms did not improve. These results suggest that HBOT might be useful for ameliorating subjective symptoms when it is administered within 3 to 4 weeks of symptom onset. We could not perform any evaluations of the "placebo effect" of HBOT because all of the patients who were admitted to our institution had been referred to us to undergo HBOT, with the aim of ameliorating their hearing loss and subjective 
symptoms. So, it would have been morally wrong not to perform HBOT in these cases.

Some studies have indicated that HBOT or treatment with steroids alone only has limited effects against acute acoustic trauma. ${ }^{8-12}$ Narozny and Conlin reported that HBOT with high doses of glucocorticoids improved the results of conventional SSHL treatment. ${ }^{13,14}$ Previous studies have shown that there are steroid receptors in the inner ear. It has been proposed that the effects of steroids are mediated through these receptors and involve the maintenance of the ion balance in the inner ear, the stabilization of cellular membranes, the enhancement of perfusion, and the inhibition of local proinflammatory cytokine expression. Salihoğlu reported that combination treatment with HBOT and steroids was not very effective against acute acoustic trauma. ${ }^{15}$ They found that $13 \%$ of patients completely recovered, whereas $\sim 11 \%$ of our patients completely recovered. In our study, the patients who were treated with a combination of HBOT, according to TT9, and steroid therapy only showed statistically significant improvements in their HPTA. Hyperbaric oxygen therapy based on TT5 did not result in significant improvements in the patients' hearing. Based on these findings, it is considered that steroids were effective when sufficient oxygen was administered during HBOT. So, it is possible that administering a combination of a sufficient amount of oxygen and steroids is important for treating acute acoustic trauma. These findings suggest that combined treatment with HBOT and steroids might be more effective against acute acoustic trauma than treatment with HBOT or steroids alone. However, this study had some limitations. For example, we should have performed an evaluation of patients who were administered steroids without HBOT to determine the utility of HBOT. However, we could not do this because only patients who are indicated for HBOT are referred to our facility.

Hyperbaric oxygen therapy can be used in cases of tinnitus in which problems during the development of the inner ear and/or brain lead to a lack of oxygen and/or limited energy provision. ${ }^{16}$ In addition, it was reported that HBOT was effective against tinnitus when administered between 2 and 6 weeks after the onset of the condition. Kuwashima performed HBOT in 37 patients with tinnitus. ${ }^{17}$ They suggested that the observed marked improvements in tinnitus were due to better inner ear microcirculation. The tinnitus seen in patients with acute acoustic trauma is probably caused by abnormal excitation of the cochlear nerve due to damage to hair cells and supporting cells resulting from impaired microcirculation. Regarding aural fullness, noise overstimulation might modulate the somatosensory ability of the tympanic membrane and change the barometric pressure in the inner ear. ${ }^{18}$ The improvements in aural fullness observed after HBOT might be due to pressure gradient correction and enhancement of the microcirculation in the inner ear.

In HBOT, 3,000 $\mathrm{L}$ and 2,500 $\mathrm{L}$ of oxygen are inhaled when treatment is administered according to TT5 and TT9, respectively, and the UPTD of TT5 and TT9 are 334 and 270, respectively. So, TT5 is considered to involve more aggressive treatment than TT9. In our study, the mean duration of the treatment period was 6.5 days and 8.5 days in the cases treated with TT5 and TT9, respectively. So, the total oxygen consumption and UPTD might have been greater in TT9, which involved a longer treatment period, than in TT5. Lafere suggested that combination treatment with aggressive, but short-term, HBOT and intravenous corticosteroids is a better option than long-term HBOT in terms of the resultant improvement in hearing ability. ${ }^{19}$ However, ignoring the effects of steroid treatment, we are not convinced that aggressive, but short-term, HBOT is superior to short-term HBOT of moderate intensity. Our study suggested that the ears that received long-term TT9-based treatment exhibited significantly greater improvements in hearing than those that received short-term TT5-based treatment $(p<0.05)$. Thus, the improvement in hearing achieved by HBOT might be influenced by the amount of oxygen inhaled or the frequency of exposure to a large amount of oxygen.

The interval from the onset of the condition to treatment was found to have little influence on whether subjective symptoms were ameliorated in the present study. Some amelioration of subjective symptoms was seen in 5 of the 11 grade 3 ears. In addition, inhaling a large amount of oxygen might help to improve subjective symptoms. There have not been any previous reports about the improvements in subjective symptoms brought about by treatment with a combination of HBOT and steroids. ${ }^{15}$ In the current study, despite there being no significant difference in the frequency of subjective symptom improvement between the cases treated with and without steroids, $\sim 85 \%$ of the patients who were treated with a combination of HBOT and steroids demonstrated improvements in their subjective symptoms, although $<25 \%$ of the patients who were treated with steroids showed improvements in their subjective symptoms before they underwent HBOT. The mean interval from the onset of the condition to the initiation of HBOT was 26.2 days among the patients whose symptoms improved, whereas it was 14.7 days among the patients whose subjective symptoms were not ameliorated. It is possible that HBOT is more effective than steroid therapy for treating acute acoustic trauma patients with subjective symptoms.

The results of this study suggest that acute acoustic trauma patients with subjective symptoms should be treated with HBOT within 3 to 4 weeks of onset, and the combined use of HBOT with steroids could be the optimal treatment for improving subjective symptoms in such cases. In addition, it might be necessary to administer a sufficient steroid dose to enhance the efficacy of such treatment before HBOT is performed for acute acoustic trauma. A randomized study of HBOT with or without steroids for acute acoustic trauma is probably required to examine these issues.

\section{Conclusion}

We evaluated the utility of HBOT as a treatment for acute acoustic trauma based on the cases treated at our institution over a 20-year period. Hyperbaric oxygen therapy should be considered as a treatment for acute acoustic trauma in cases 
involving subjective symptoms, such as tinnitus and aural fullness.

\section{Conflict of Interest}

The authors have no conflicts of interest that are directly relevant to the content of this article.

\section{References}

1 Bapat U, Tolley N. Temporary threshold shift due to recreational firearm use. J Laryngol Otol 2007;121(10):927-931

2 Quaranta A, Portalatini P, Henderson D. Temporary and permanent threshold shift: an overview. Scand Audiol Suppl 1998; 48:75-86

3 Counter SA, Klareskov B. Hypoacusis among the Polar Eskimos of northwest Greenland. Scand Audiol 1990;19(03):149-160

4 Olszewski J, Miłoński J, Olszewski S, Majak J. Hearing threshold shift measured by otoacoustic emissions after shooting noise exposure in soldiers using hearing protectors. Otolaryngol Head Neck Surg 2007;136(01):78-81

5 Harada H, Shiraishi K, Kato T, Sota T. Acute Acoustic Trauma of Self Defense Force Officials. Audiol Jpn 1992;35:173-177(in Japanese)

6 Matsunobu T. Noise induced sudden hearing loss. Otolaryngology-Head and Neck Surgery (Tokyo) 2015;87(08):594-601(in Japanese)

7 Ylikoski J, Mrena R, Makitie A, Kuokkanen J, Pirvola U, Savolainen S. Hyperbaric oxygen therapy seems to enhance recovery from acute acoustic trauma. Acta Otolaryngol 2008;128(10):1110-1115

8 Colombari GC, Rossato M, Feres O, Hyppolito MA. Effects of hyperbaric oxygen treatment on auditory hair cells after acute noise damage. Eur Arch Otorhinolaryngol 2011;268(01):49-56
9 d'Aldin C, Cherny L, Devrière F, Dancer A. Treatment of acoustic trauma. Ann N Y Acad Sci 1999;884:328-344

10 Fakhry N, Rostain JC, Cazals Y. Hyperbaric oxygenation with corticoid in experimental acoustic trauma. Hear Res 2007;230 (1-2):88-92

11 Harada H, Ichikawa D, Imamura A. Course of hearing recovery according to frequency in patients with acute acoustic sensorineural hearing loss. Int Tinnitus J 2008;14(01):83-87

12 van der Veen EL, van Hulst RA, de Ru JA. Hyperbaric Oxygen therapy in acute acoustic trauma: a rapid systematic review. Otolaryngol Head Neck Surg 2014;151(01):42-45

13 Narozny W, Sicko Z, Przewozny T, Stankiewicz C, Kot J, Kuczkowski J. Usefulness of high doses of glucocorticoids and hyperbaric oxygen therapy in sudden sensorineural hearing loss treatment. Otol Neurotol 2004;25(06):916-923

14 Conlin AE, Parnes LS. Treatment of sudden sensorineural hearing loss: I. A systematic review. Arch Otolaryngol Head Neck Surg 2007;133(06):573-581

15 Salihoğlu M, Ay H, Cincik H, et al. Efficiency of hyperbaric oxygen and steroid therapy in treatment of hearing loss following acoustic trauma. Undersea Hyperb Med 2015;42(06):539-546

16 Böhmer D. Treating Tinnitus with Hyperbaric Oxygenation. Int Tinnitus J 1997;3(02):137-140

17 Kuwashima S, Ishijima K, Sato H, Murai K. Forefront of tinnitus therapy: Hyperbaric oxygen therapy. MB ENT 2005;49:81-85(in Japanese)

18 Sakata T, Higuchi H, Ueno T, Nakagawa T. Modulation of somatosensory abilities and the feeling of ear fullness in patients with acute sensorineural hearing loss. Auris Nasus Larynx 2012;39 (03):265-269

19 Lafere P, Vanhoutte D, Germonpe P. Hyperbaric oxygen therapy for acute noise-induced hearing loss; evaluation of different treatment regimens. Diving Hyperb Med 2010;40:63-67 